\author{
Ewa Stala \\ Uniwersytet Jagielloński w Krakowie \\ ewa.stala@uj.edu.pl
}

\title{
Los diccionarios de P. S. Pallas y Ph. A. Nemnich Hallazgos y enigmas
}

\section{Resumen:}

El objetivo del trabajo es presentar dos fuentes lexicográficas relativamente poco conocidas (al menos en el entorno hispanista) que contienen material español: Linguarum totius Orbis vocabularia comparativa, también llamado «Diccionario de Catalina la Grande» (1787-1789), de Peter Simon Pallas, y Waaren-Lexicon enzwölf Sprachen (1797), de Philipp Andreas Nemnich. Ambas obras fueron publicadas a finales del siglo XVIII, fuera de España, y sus autores son dos científicos alemanes (un biólogo y un abogado). El presente análisis se inscribe dentro de la llamada «lingüística de corpus» y el resultado son nuevos hallazgos en el vocabulario español: antedataciones, nuevas variantes, nuevas formas o afiliación de los dos diccionarios.

Palabras clave: Diccionario de Catalina la Grande, Waaren-Lexicon, lexicografía, diacronía, español

\begin{abstract}
:
The Dictionaries of P. S. Pallas and Ph. A. Nemnich. Findings and Enigmas The main aim of the study is to present two lexicographic sources which contain Spanish lexical material and remain relatively unknown in the Spanish context which contain Spanish lexical material, namely Linguarum totius Orbis vocabularia comparativa, also called Dictionary of Catherine the Great (1787-1789), by Peter Simon Pallas, and Waaren-Lexicon in zwölf Sprachen (1797), by Philipp Andreas Nemnich. Both works were published in late $18^{\text {th }}$ century, outside Spain, and their authors are two German scholars (a biologist and a lawyer). The present
\end{abstract}


analysis uses the methodology corpus linguistics and it contributes new findings in the area of Spanish vocabulary: antedatings, new variants, new forms as well as the affiliation of the two dictionaries.

Keywords: Dictionary of Catherine the Great, Waaren-Lexicon, lexicography, diachrony, Spanish

Ambos diccionarios los encontré gracias a la gentileza del Prof. Stanisław Stachowski, turcólogo y eslavista a la vez. Ambos, al mismo tiempo, se encuentran en bibliotecas de Cracovia. Otra coincidencia es que fueron publicados casi simultáneamente: el lexicón de Nemnich, en 1797; el de Pallas, entre 1787 y 1789. Aunque los dos autores son alemanes, sus vidas y carreras difieren bastante: de ahí que primero hagamos un esbozo biográfico de cada uno de ellos. Un análisis detallado de las obras se halla en sendas publicaciones (Stala, 2011, 2015); aquí solo presentamos, tal como indica el título, nuevos hallazgos y algunas incógnitas que, esperamos, inciten a nuevas investigaciones.

Peter Simon (Pedro Simón) Pallas, naturalista alemán (1741-1811), estudió Medicina en Berlín y Gotinga para, finalmente, dedicarse a las ciencias naturales. En 1760 se trasladó a la Universidad de Leiden y con diecinueve años presentó una tesis doctoral que trataba sobre la fauna intestinal. En 1767 fue invitado por Catalina II de Rusia a la Academia de Ciencias de San Petersburgo, de la que fue nombrado miembro adjunto; a partir de 1768, ejerció como profesor de la Universidad de Petersburgo. Entre 1769 y 1774 dirigió una expedición a Siberia, donde recogió numerosos especímenes. En 1777 fue designado miembro de la oficina topográfica rusa y en los años 1793-94 se puso a la cabeza de una segunda expedición al sur de Rusia, durante la cual visitó Crimea y el Mar Negro. Después de treinta años de trabajo, la zarina lo nombró historiador del Almirantazgo y profesor del heredero del trono, ofreciéndole terrenos en Crimea. Pallas dejó más de cuarenta obras sobre etnografía, paleontología, zoología, geología, botánica, geografía, más allá de los diccionarios y memorias. Entre ellos, se halla una obra de carácter lexicográfico: Linguarum totius 
Orbis vocabularia comparativa, cuyo título completo es: Linguarum totius Orbis vocabularia comparativa. Augustissime cura collecta. Sectio prima, lenguas Europae et Asiae complexa. Petropoli, Typis Iohannis Caroli Schnoor, 1787-1789. En realidad, no hay unanimidad en cuanto a la fecha de edición del primer volumen de la obra: aunque en la portada de la versión de Wendland (1991) figura el año 1787, otras fuentes (Triana y Antorveza, [on-line]; Niederehe, Koerner, 1990) mencionan el año 1786. La obra de Pallas ha sido estudiada por varios investigadores (Juszkiewicz, 1975, 1976; Stachowski, 1987; Popowska-Taborska, 1994, 2006; Fałowski, 1999a, 1999b, 2000; Bochnakowa, 2000, 2013; Zulaika, 2009; Stala, 2011; Igartua, 2012; Jakubczyk, 2014a, 2014b).

La versión que hemos manejado ${ }^{1}$ contiene, entre otras, lenguas eslavas, románicas y germánicas junto con el griego, el albanés, el gitano, el chino, el japonés, el malasio, el siamés o la lengua vangoo. En cuanto a la macroestructura del diccionario, el punto de partida es el ruso. A esta entrada le siguen los equivalentes en los idiomas numerados de 1 (eslavo) a 200 (Islas Sandwich, actualmente Islas Hawái). Las entradas, ordenadas, bastante libremente, según la temática mencionada más detalladamente en la parte analítica, suman un total de $272^{2}$ [tomo 1: 1-130, tomo 2: 131-272]. El número 25 de cada entrada pertenece al español. Lo más curioso es que todo el diccionario ha sido redactado en alfabeto cirílico, lo que claramente refleja la idea que se tenía en el s. XVIII de un alfabeto universal.

Respecto a la microestructura, la unidad lexicográfica de referencia es la palabra. Las entradas aparecen en nominativo singular en el caso de los sustantivos; en nominativo singular masculino las de los adjetivos, y en infinitivo las de los verbos. Generalmente, aparece un equivalente en cada uno de los idiomas, aunque a veces se recogen dos (cf. fogon, hogar [фогон, ғогаръ] - focus; pendencia, contienda [пенденцїя, контїенда] - rixa; bárca, náve [бáрка, на́ве] - navis;

${ }^{1}$ Signatura en la Biblioteca del Instituto de Filología Eslava Oriental de la Universidad Jaguellónica: 4996/1/O (t. 1), 4996/2/O (t. 2).

${ }^{2}$ Al menos en la edición aquí manejada; Igartua (2012) menciona 285. 
pequeno, poco [пекено, поко] - parvum; llevar, traer [льеваръ, траерь - vehere. Pocas veces el mismo vocablo español equivale a dos entradas diferentes: poder [подеръ] - potentia; poder [подеръ] - potestas. Los principales campos léxicos son: la vida cotidiana, los fenómenos de la naturaleza, las partes del cuerpo, los cromatónimos, la flora, la fauna y otros (Juszkiewicz, 1976). Los aspectos analíticos que se recogen son: la transliteración, la fonética, la morfología y el semantismo de las formas documentadas ${ }^{3}$.

En conjunto, en lo que concierne al material español, lo que suscita más interés son:

- los arcaísmos:

- (24) fronte [фронте] - frente; por haber mantenido la consonante final o la vocal postónica final;

- (259) non [нонь] - no;

- (179) pane [пане] - pan; esta última una posible ultracorrección;

- los vocablos desusados o sustituidos actualmente por otros:

- (163) anade [анаде] - anas; actualmente ánade 'pato, ave con los mismos caracteres genéricos que el pato' (DRAE); de alta frecuencia de uso en los ss. XIV-XV (v. CORDE);

- (187) malhora [малғора] - miseria; actualmente solo un coloquialismo propio del español de México 'amigo de hacer maldades o travesuras' (DRAE, 1992: 918); documentado en el CORDE en: c. 1550, 1561, 1602, c. 1605.

- (226) regociado [регоциадо] 'contento'; hoy: regocijado, no se documenta en el CORDE.

- las variantes fonéticas o gráficas no encontradas en ninguna de las fuentes lexicográficas consultadas (Tesoro Lexicográfico, CORDE, etc.):

- (32) pescuso [пескуссо] - collum;

- (183) curaça [кураца] - thorax;

- (127) ierva [иерба] - hierba; está documentado en los diccionarios de Nebrija (1492), Covarrubias (1611) o Minsehu (1617).

${ }^{3}$ Sobre lo problemático que puede resultar dicha transliteración, v. Bochnakowa (1999). 
- los lexemas no documentados en ninguna de las fuentes consultadas:

- (91) etades [этадесъ] - aestas 'verano'; probablemente acus. del lat. aestas, -tis 'íd.';

-(19) ventas [вентась] - nares 'nariz'; probablemente tiene que ver con ventana 'cada uno de los orificios de la nariz' < lat. ventus;

- (173) medino [медино] - mensura; probablemente, extensión del significado concreto 'moneda de cobre usada en Turquía' también denominada para, palabra esta derivada del persa pārah, lit. 'trozo'4;

- (214) legero [легеро] - laevis; esta variante de la forma actual ligero fue documentada, según nuestros conocimientos, en tan solo un diccionario: Vocabularium Hispanicum Latinum et Anglicum copiosissimum [...], editado en Londres en 1617 y cuyo autor es John Minsehu (legero 'vid. Ligéro'). Una de las posibles vías de trasvase es la amistad que Pallas trabó con el anticuario y naturalista galés Thomas Pennant (1726-1798), con quien mantuvo una abundante correspondencia.

El lexicón de mercancías de Ph. A. Nemnich es prácticamente desconocido en el mundo hispano $0^{5}$, en general, no cuenta con un número elevado de análisis. Han sido realizados los del léxico ruso (Fałowski, 2008) y el español (Stala, 2015). La obra en cuestión fue publicada en Hamburgo en 1797 bajo título: Waaren-Lexicon in zwölf Sprachen der hamburgischen Commerz-Deputation zugeeignet; y fue digitalizada en 2009 por la Biblioteca Estatal de Baviera. Su autor, Philipp Andreas Nemnich (1764-1822), como Pallas, fue abogado, pero trabajó también para Allgemeine Zeitung y así pudo viajar por toda Europa. Es autor de once obras de carácter principalmente lexicográfico y de diarios de

${ }^{4}$ Medino, sinónimo de para "a former copper coin of Turkey, the 40th part of piaster; 1680-90< Turk < pers. pārah, lit. piece" (http://dictionary.reference. $\mathrm{com} /$ browse/Para).

${ }^{5}$ El compendio Lexicografía española (Medina Guerra, 2003) menciona tan solo una obra de Nemnich: Lexicon Nosologicum polyglotton (1793-1798). 
viajes. Waaren-Lexicon ${ }^{6}$ consta de dos partes: la primera (pp. 1-424) la forman once glosarios bilingües, cuyas segundas mitades son siempre en alemán. Las lenguas de las primeras mitades son: inglés, holandés, sueco, danés, francés, italiano, español, portugués, ruso, polaco y latín:

Fideos. Nudeln.

Madre perla. Perlenmutter.

La segunda parte (pp. 424-574) lleva por título Deutches Waaren-Lexicon gleichbedeutenden holländischen, dänischen, schwedischen, englischen, französischen, italienischen, spanischen, portugiesischen, russischen, polnischen, und lateinischen Benennungen y contiene lemas alemanes, ordenados alfabéticamente, más sus equivalentes en los otros idiomas:

Mundleim.

H. Mondlym.

D. Mundlim.

S. Munlim.

E. Mouth glue, Lip-glue.

F. Colle a bouche.

I. Colla da bocca.

S. Cola de boca.

P. Cola de boca.

El objeto de nuestro análisis ha sido el material español recogido en la primera parte: Spanisches Waaren-Lexicon (3127 lemas). Asimismo, las unidades de la segunda parte (Deutsches Waaren-Lexicon) aparecen mayoritariamente ya en la primera (Spanisches Waaren-Lexicon). A veces, Nemnich en vez del equivalente alemán propone uno en francés o en latín:

Palma Christi. El Ricino vulgar.

Vizcochos. Los Bizcochos.

Anascote. Serge d'ascot.

${ }^{6}$ Hemos manejado el ejemplar de la Biblioteca Jaguellónica (signatura 586259 I Mag. St. Dr.). 
Polvos de soconusco. Pulvis indicus aromaticus.

Asombra la riqueza léxica de la obra y el profundo conocimiento de las mercancías (numerosos tipos de aceite, azúcar, telas, etc.). En la introducción el autor menciona a las personas relacionadas directamente con el comercio; no alude, sin embargo, a las fuentes de su obra. Tras un análisis pormenorizado, se puede suponer que en gran parte se sirvió del Diccionario de Autoridades (1726-39) y del Diccionario castellano con las voces de ciencias y artes y sus correspondientes en las tres lenguas francesa, latina e italiana (17861793), de un jesuita español, Esteban Terreros y Pando. Los campos léxicos comprenden: nombres del mundo animal y vegetal, especies de madera, telas, elementos de la vida cotidiana, herramientas, armas, metales, minerales, sustancias químicas o instrumentos musicales. El análisis, igual que en el caso del repertorio de Pallas, abarca la grafía, la fonética, la morfología y la semántica de las voces, y también algunos descubrimientos del área del léxico español. Esos últimos son, al parecer, bastante numerosos. Baste con decir que hemos sido capaces de antedatar 109 lexemas:

- agebe 1895

- alambrillo 1853

- alana 1853

- alemaneta 1844

- amster 1853

- arlequin 1878

- arraclan 1817

- baroto 1798

- betulla 1942

- bucio 1853

- cabilla 1803

- cabillon 1852

- cambrayuelo 1936

- caña de Indias 1997

- caput mortuum 1809
- castorcillo 1803

- caviario 1853

- cepillito 1853

- chalote 1822

- chancaca 1846

- chavasca 1914

- chitan 1853

- cholet 1895

- cochehue 1805

- colinabo 1846

- colmenilla 1825

- copalchi 1812

- coye 1860

- cretona 1853

- cubrecama 1846 
- damesana 1918

- diente de lobo 1933-46

- dragoncillo 1853

- equiseto 1853

- escarlatilla 1853

- escarminador 1836

- esparcilla 1825

- estanquia 1831

- fajol 1917

- ficha 1803

- frejol 1817

- frijon 1895

- galipodio 1825

- garrofe 1951

- glaseado 1803

- goma-guta 1853

- guyana 1853

- hachita 1853

- hipocistidos 1855

- hueso del corazon del ciervo 1839

- indiana de algodon 1803

- jibion 1817

- lasqui 1816

- licopodio 1846

- limpia(-)oídos 1846

- liron 1895 ('planta')

- lobogante 1862

- loneta 1853

- magarzuela 1822

- manatin 1969

- manganesia 1832

- manquerna 1808

- marun 1808

- mermolada 1829
- moleton 1825

- moscovia 1854

- murgura 1895

- negro de hueso 1878

- negro de marfil 1822

- oreja de Judas 1803

- organsino 1855

- palo de Pernambuco 1814

- paloduz 1947

- palomillo 1822

- pantufla 1874

- papel para imprimir 1884

- pasabalas 1846

- pataca 1803

- peigne 1942

- pezpalo 1843

- prusiana 1895

- quassia s. XX

- rinconera 1803

- roldon 1925

- rom 1853

- ron 1803

- sacacorchos 1803

- sacatrapo 1872

- safio 1803

- sangueso 1843

- sanguiñol 1860

- semencontra 1855

- silesia 1851-58

- sobrebueno 1855

- sopera 1803

- suavizador 1803

- tacuache 1917

- tapaculo 1803

- tierra japonica 1803 
- tolmeza 1808

- unicornio marino 1857

- vaca marina 1832

- vencetosigo 1853

- verimbao 1809
- veza 1884

- zafranon 1808

- zafre 1817

- zink 1855

- yebo 1925.

Hemos descubierto 38 nuevas variantes de formas ya conocidas:

- ajonjole act. ajonjolí

- alledano act. aledano

- almanake act. almanaque

- altaracea act. taracea

- arcaneta act. orcaneta

- asabacar act. asarabácara

- brim act. brin

- cadir act. cádiz

- cadizo act. cádiz

- cameloncillos act. camelotillos

- cañaveta act. cañivete

- castorino act. castorina

- cocolmeca act. cozolmeca

- comote act. camote

- couti act. cutí

- criadillo act. criadilla

- ederdon act. edredón

- esparceta act. esparseta

- galgano act. galangal

- goma armoniaco act. (goma) amoniaco
- granite act. granito

- jenista act. genista

- malaqui act. malaquita

- malaquite act. malaquita

- millepedes act. milpiés

- nanquina act. nanquín

- pincela act. pincel

- principelilla act. principela

- orcanete act. orcaneta

- reseco act. reseca

- rulda act. roldón

- sago act. sagú

- sesame act. sésamo

- seselio act. seselí

- tabarete act. taburete

- tapo act. topo

- (trigo) saraceno act. (trigo) sarraceno

- vermillon act. vermellón.

Una atención especial merecen las nuevas acepciones de algunos lexemas conocidos:

- azor 'especie de lino'

- casero 'paño hecho en casa' 
- enjeño 'espátula para dar la vuelta al asado'

- liron 'especie de máquina'

- mano de Judas 'cierta especie de matacandelas, en forma de mano'

- mufla 'concha'

- rehilada prob. 'hilo rehilado'

- reseco 'especie de lana'

- volante 'planta acuática'.

Aunque hemos sido capaces de descubrir la etimología de 34 unidades léxicas: acajou, albiña, agra, alemaneta, ambarilla, antolaje, apocino, bondon, calafraga, catapucia, cipero, durancillo, dutroa, espodio, estanquia, esteca, esrecado, gusa, guyana, harderic, macio, malabatro, matalaz, oblon, oleandro, pamplemusa, pareira brava, pizacanta, politrico, rodulo, sangueso, seseli, tamalapatra, vejin, yusquiamo, las siguientes etimologías esperan su solución:

- almangre

- alpibre

- archenda

- arlet

- baroto

- bimaes

- bova
- bucela

- caga

- cochehue

- enjeño

- ganzul

- gengiba

- jiraspe
- joyo-lino

- lacargama

- lasqui

- pinsbech

- saidalla

- segurela

- tarajaco.

Y, lo más interesante, en el repertorio de Nemnich se hallan tres formas que pueden considerarse hapax, no documentadas en ninguna otra fuente:

- cegueries 'piel de marta';

- durancillo 'especie de paño';

- saidalla 'alamar'7.

7 Todos estos significados son reflejo del de los equivalente alemanes indicados por Nemnich. 
Tal como se desprende del estudio presentado, el léxico español sigue sorprendiendo a la hora de descubrir nuevos documentos. Obviamente, son formas de escasa penetración en español, pero al haber sido documentadas en glosarios plurilingües, indudablemente forman parte de la historia del léxico español. Quedan por descubrir tanto las voces mencionadas arriba como las de otros varios idiomas. Es más, el acceso a ambas obras ha sido facilitado gracias a sus versiones digitalizadas, una clara invitación a futuros análisis y nuevos hallazgos.

\section{Bibliografía}

BOCHNAKOWA, A. (1999/2000), "Francuskie wyrazy w XVIII-wiecznym słowniku porównawczym Linguarum totius orbis vocabularia comparativa (1787-1789)", Sprawozdania z Czynności i Posiedzeń Polskiej Akademii Umiejętności, 63, Kraków, pp. 109-112.

BOCHNAKOWA, A. (2013), "«Linguarum totius orbis Vocabularia comparativa Augustissimae cura collecta (...)», 1787-1789”, Studia Linguistica Universitatis Iagellonicae Cracoviensis, 129(1), Kraków, pp. 41-50, [on-line] https://doi.org/10.4467/20834624SL.13.002.1133 .

FAŁOWSKI, A. (1999a), "Materiał połabski w Słowniku Katarzyny II”, en: Brzezina, M., Kurek, H. (red.), Collectanea linguistica in honorem Casimiri Polański, Księgarnia Akademicka, Kraków, pp. 49-57.

FAŁOWSKI, A. (1999b), “Język ukraiński w Słowniku Katarzyny II”, en: Mieczkowska H. (red.), In Memoriam Alfredi Zaręba et Josephi Reczek, Wydawnictwo Oddziału Polskiej Akademii Nauk, Kraków, pp. 137-144. FAŁOWSKI, A. (2000), "Materiał czeski w Słowniku Katarzyny II", en: Wróbel H. (red.), Studia z filologii słowiańskiej ofiarowane profesor Teresie Zofii Ortoś, Wydawnictwo Uniwersytetu Jagiellońskiego, Kraków, pp. 89-94.

FAŁOWSKI, A. (2008), Stownictwo rosyjskie w Waaren-Lexicon in zwölf Sprachen Ph. A. Nemnicha (Hamburg 1789), Wydawnictwo Uniwersytetu Jagiellońskiego, Kraków.

IGARTUA, I. (2012), "Las fuentes lexicográficas vascas en el vocabulario políglota de P. S. Pallas”, en: Battaner Moro, E., Calvo Fernández, V., 
Peña Jiménez, P. (eds.), Historiografía lingüistica: líneas actuales de investigación, vol. 2, Nodus, Münster, pp. 485- 496.

JAKUBCZYK, M. (2014a), "Polszczyzna grażdańska w słowniku carycy Katarzyny II", Poradnik Językowy, 2, pp. 78-95.

JAKUBCZYK, M. (2014b), "Słownik carycy Katarzyny Wielkiej w kontekście europejskich teorii językowych II połowy XVIII wieku i na tle tradycji leksykograficznej”, Poradnik Językowy, 9, pp. 64-77.

JUSZKIEWICZ, U. (1975), "Porównawczy słownik Katarzyny II na tle rozwoju rosyjskiego języka literackiego XVIII wieku", Annales Universitatis Mariae Curie-Skłodowska. Sectio F, Nauki Humanistyczne, 30, pp. 157-168.

JUSZKIEWICZ, U. (1976), "Materiał rosyjski ze słownika Katarzyny II", Annales Universitatis Mariae Curie-Skłodowska. Sectio F, Nauki Humanistyczne, 31, pp. 385-401.

LARRUCEA DE TOVAR, C. (1984), “José Celestino Mutis (1732-1808) and His Report on American Languages Ordered by Charles III of Spain for Catherine the Great of Russia", Historiographia Linguistica, 11(1-2), pp. 213-229.

MEDINA GUERRA, M. A. (ed.) (2003), Lexicografía española, Ariel, Barcelona.

NEMNICH, Ph. A. (1797), Waaren-Lexicon in zwölf Sprachen der hamburgischen Commerz-Deputation zugeeignet, Hamburg.

NIEDEREHE, H. J., KOERNER, E. F. K. (eds.) (1990), History and Historiography of Linguistics, vol. 2: 18th-20th Century, John Benjamins, Amsterdam, [on-line] https://doi.org/10.1075/sihols.51.2.

PALLAS, P. S. (1787-1789), Сравнительные словари встхъ языковъ и наргиій... Linguarum totius orbis vocabularia comparativa, 17871789 , t. 1-2, Petersburg.

POPOWSKA-TABORSKA, H. (1994), "Słownictwo kaszubskie w osiemnastowiecznych porównawczych słownikach języków Europy i Azji”, Rocznik Slawistyczny, 49, pp. 41-46.

POPOWSKA-TABORSKA, H. (2006), "Raz jeszcze o materiałach kaszubskich w «Słownikach porównawczych języków i narzeczy 
Europy i Azji»", Szkice, pp. 287-296 [1ª ed.: Rocznik Gdański 1998, 58(1), Gdańsk, pp. 163-171].

STACHOWSKI, S. (1987), "Wyrazy albańskie w Słowniku P. S. Pallasa (1787-1789)", Acta Baltico-Slavica, 17, Warszawa, pp. 237-253.

STALA, E. (2011), "Diccionario de Catalina la Grande (1787-1789). Análisis del material español”, Studia Iberystyczne, 10, Kraków, pp. 151-163.

STALA, E. (2015), El léxico español en el Waaren-Lexicon in zwölf Sprachen de Ph. A. Nemnich, Peter Lang Edition, Frankfurt am Main, [on-line] http://dx.doi.org/10.3726/978-3-653-05439-2.

WENDlAND, F. (1991), Peter Simon Pallas (1741-1811), Mouton de Gruyter, Berlin-New York, [on-line] https://doi.org/10.1515/978 3110853445.

ZULAIKA HERNÁNDEZ, J. M. (2009), “El euskera en la obra de Edward Lhuyd (1660-1709)”, Oihenart, 24, pp. 291-334.

\section{Enlaces de Internet}

Diccionario de la Real Academia Española (1992), ed. 21'a , [on-line] http:// buscon.rae.es/, 17.06.2015.

http://dictionary.reference.com/browse/Para, [on-line] 20.06.2015

Real Academia Española, Banco de datos (CORDE), [on-line] Corpus diacrónico del español. http://www.rae.es, 14.06.2015.

TRIANA Y ANTORVEZA, H., Las lenguas indígenas en el ocaso del Imperio Español, [on-line] www.philosophia.cl/ Escuela de Filosofía Universidad ARCIS, 9.06.2011. 